\title{
Stabilization of the Wave Equation with Boundary Time-Varying Delay
}

\author{
Hao Li, ${ }^{1}$ Changsong Lin, ${ }^{1}$ Shupeng Wang, ${ }^{2}$ and Yanmei Zhang ${ }^{3}$ \\ ${ }^{1}$ School of Ocean Sciences, China University of Geosciences, Beijing 100083, China \\ ${ }^{2}$ Institute of Information Engineering, Chinese Academy of Sciences, Beijing 100093, China \\ ${ }^{3}$ School of Information Engineering, China University of Geosciences, Beijing 100083, China
}

Correspondence should be addressed to Hao Li; lihaopaper@sohu.com

Received 18 October 2013; Revised 10 January 2014; Accepted 10 January 2014; Published 26 February 2014

Academic Editor: Wen-Xiu Ma

Copyright (C) $2014 \mathrm{Hao}$ Li et al. This is an open access article distributed under the Creative Commons Attribution License, which permits unrestricted use, distribution, and reproduction in any medium, provided the original work is properly cited.

We study the stabilization of the wave equation with variable coefficients in a bounded domain and a time-varying delay term in the time-varying, weakly nonlinear boundary feedbacks. By the Riemannian geometry methods and a suitable assumption of nonlinearity, we obtain the uniform decay of the energy of the closed loop system.

\section{Introduction}

Let $\Omega$ be a bounded domain in $\mathbb{R}^{n}$ with smooth boundary $\Gamma$. It is assumed that $\Gamma$ consists of two parts $\Gamma_{1}$ and $\Gamma_{2}\left(\Gamma=\Gamma_{1} \cup \Gamma_{2}\right)$ with $\Gamma_{2} \neq \emptyset, \bar{\Gamma}_{1} \cap \bar{\Gamma}_{2}=\emptyset$. Define

$$
\mathscr{A} u=-\operatorname{div} A(x) \nabla u \text { for } u \in H^{1}(\Omega),
$$

where div is the divergence operator of the standard metric of $\mathbb{R}^{n} . A(x)=\left(a_{i j}(x)\right)$ is symmetric, positively definite matrices for each $x \in \mathbb{R}^{n}$ and $a_{i j}(x)$ are smooth functions on $\mathbb{R}^{n}$.

We consider the stabilization of the wave equations with variable coefficients and time-varying delay in the dissipative boundary feedback as follows:

$$
\begin{gathered}
u_{t t}+\mathscr{A} u=0 \quad(x, t) \in \Omega \times(0,+\infty), \\
\left.u(x, t)\right|_{\Gamma_{2}}=0 \quad t \in(0,+\infty), \\
\frac{\partial u(x, t)}{\partial v_{\mathscr{A}}}+\phi(t)\left(\mu g_{1}\left(u_{t}(x, t)\right)+\lambda g_{2}\left(u_{t}(x, t-\tau(t))\right)\right)=0 \\
(x, t) \in \Gamma_{1} \times \in(0,+\infty), \\
u(x, 0)=u_{0}(x), \quad u_{t}(x, 0)=u_{1}(x) \quad x \in \Omega, \\
u_{t}(x, t-\tau(0))=f_{0}(x, t-\tau(0)) \quad(x, t) \in \Gamma_{1} \times(0, \tau(0)),
\end{gathered}
$$

where $\tau(t)$ satisfies

$$
0 \leq \tau(t) \leq \tau_{0}, \quad d_{0} \leq \tau^{\prime}(t) \leq d<1 \quad \forall t \geq 0,
$$

where $\tau_{0}>0$ and $d_{0}$ and $d$ are constants. $g_{1} \in C(\mathbb{R})$ and there exist positive constants $c_{1}, p \geq 1$ such that

$$
\begin{gathered}
g_{1}(0)=0, \quad s g_{1}(s) \geq|s|^{2} \quad \text { for } s \in \mathbb{R}, \\
\left|g_{1}(s)\right| \leq c_{1}|s| \quad \text { for }|s|>1, \\
s^{2}+\left(g_{1}(s)\right)^{2} \leq c_{1}\left(s g_{1}(s)\right)^{1 / p} \text { for }|s| \leq 1 .
\end{gathered}
$$

$g_{2}(s) \in C(\mathbb{R})$ satisfies

$$
\left(g_{2}(s)\right)^{2} \leq s g_{1}(s) \quad \text { for } s \in \mathbb{R},
$$

and $\phi(t) \in C([-\tau(0),+\infty))$ satisfies

$$
\begin{gathered}
0<\phi(t) \leq \phi_{0} \quad \forall t \geq-\tau(0), \\
c_{2} \phi(t) \leq \phi(t-\tau(t)) \leq c_{3} \phi(t) \quad \forall t \geq 0, \\
\lim _{t \rightarrow \infty} \frac{F(t)}{t}=0,
\end{gathered}
$$

where $\phi_{0}, c_{2}$, and $c_{3}$ are positive constants and $F(t)=$ $1 / \inf \{\phi(\rho) \mid 0 \leq \rho \leq t\}$. 
$\partial u / \partial v_{\mathscr{A}}$ is the conormal derivative

$$
\frac{\partial u}{\partial v_{\mathscr{A}}}=\langle A(x) u, v\rangle
$$

where $\langle\cdot, \cdot\rangle$ denotes the standard metric of the Euclidean space $\mathbb{R}^{n}$ and $\nu(x)$ is the outside unit normal vector for each $x \in \Gamma$. Moreover, $\mu>0, \lambda \in \mathbb{R}, \lambda \neq 0$, and the initial data $\left(u_{0}, u_{1}, f_{0}, w_{0}, w_{1}, h_{0}\right)$ belongs to a suitable space.

There is a specific example for $\phi(t)$. Let $\phi_{1}>0$ be a constant. If $\phi(t)$ satisfies

$$
\phi_{1} \leq \phi(t) \leq \phi_{0} \quad \forall t \geq 0,
$$

then

$$
\begin{gathered}
\frac{\phi_{1}}{\phi_{0}} \phi(t) \leq \phi(t-\tau(t)) \leq \frac{\phi_{0}}{\phi_{1}} \phi(t) \quad \forall t \geq 0, \\
\lim _{t \rightarrow \infty} \frac{F(t)}{t} \leq \frac{\phi_{0}}{t}=0 .
\end{gathered}
$$

Conditions (7) and (8) hold.

In absence of delay $(\lambda=0)$, the problem (2) was studied by [1-8] and many others. The decay rate of the energy (when $t$ goes to infinity) depends on the function $\phi$ and the growth of $g_{1}$.

The system (2) with constant coefficient (the case: $A(x)$ is a constant matrix on $\bar{\Omega}$ ) was studied by [9-11] and many other authors. For the system (2) with variable coefficients, the main tools to cope with the system (2) are the differential geometrical methods which were introduced by [12] and have been applied in many papers. See [13-22] and references cited therein. For a survey on the differential geometric methods, see $[23,24]$.

The authors in [11] considered the system (2) with constant coefficients operator and dissipative boundary conditions of time dependent delay and proved the exponential decay of the energy by combining the multiplier method with the use of suitable integral inequalities. Different from this paper, $g_{1}$ is assumed to be linearly bounded and $\phi$ is assumed to be a constant function in the paper [11].

Based on [11], the purpose of this paper is to solve the stability of the system (2) with variable coefficients and timevarying, weakly nonlinear terms. To obtain our stabilization result, we assume that

$$
\left(\frac{1}{2 c_{2}}+\frac{1}{2}\right) \frac{|\lambda|}{\sqrt{1-d}}<\mu,
$$

where $c_{2}$ is defined in (8).

Define the energy of the system (2) by

$$
\begin{aligned}
E(t)= & \frac{1}{2} \int_{\Omega}\left(u_{t}^{2}+\sum_{i, j=1}^{n} a_{i j} u_{x_{i}} u_{x_{j}}\right) d x \\
& +\xi \int_{t-\tau(t)}^{t} \int_{\Gamma_{1}} \phi(\rho) u_{\rho}(x, \rho) g_{1}\left(u_{\rho}(x, \rho)\right) d \Gamma d \rho,
\end{aligned}
$$

where $\xi$ is a positive constant satisfying

$$
\frac{|\lambda|}{2 c_{2} \sqrt{1-d}}<\xi<\mu-\frac{|\lambda|}{2 \sqrt{1-d}} \text {. }
$$

We define

$$
g=A^{-1}(x) \quad \text { for } x \in \mathbb{R}^{n}
$$

as a Riemannian metric on $\mathbb{R}^{n}$ and consider the couple $\left(\mathbb{R}^{n}, g\right)$ as a Riemannian manifold with an inner product

$$
\langle X, Y\rangle_{g}=\left\langle A^{-1}(x) X, Y\right\rangle, \quad|X|_{g}^{2}=\langle X, X\rangle_{g} \quad X, Y \in \mathbb{R}_{x}^{n} .
$$

Let $D_{g}$ denote the Levi-Civita connection of the metric $g$. For the variable coefficients, the main assumptions are as follows.

Assumption $A$. There exists a vector field $H$ on $\bar{\Omega}$ and a constant $\rho_{0}>0$ such that

$$
D_{g} H(X, X) \geq \rho_{0}|X|_{g}^{2} \quad \text { for } X \in \mathbb{R}_{x}^{n} \quad x \in \bar{\Omega} .
$$

Moreover, we assume that

$$
\begin{gathered}
\sup _{x \in \bar{\Omega}} \operatorname{div} H<\inf _{x \in \bar{\Omega}} \operatorname{div} H+2 \rho_{0}, \\
H \cdot v \leq 0 \quad x \in \Gamma_{2}, \quad H \cdot v \geq \delta \quad x \in \Gamma_{1},
\end{gathered}
$$

where $\delta$ is a positive constant.

Assumption (17) was introduced by [12] as a checkable assumption for the exact controllability of the wave equation with variable coefficients. Assumption A is also useful for the controllability and the stabilization of the quasilinear wave equation [15]. For the examples of the condition, see [12, 23].

Based on Assumption (17), Assumption A was given by [22] to study the stabilization of the wave equation with variable coefficients and boundary condition of memory type. The authors in [22] also constructed some examples of the condition based on the assumption that $A(x)=a(x) I$ or $A(x)$ is a perturbation of a symmetric positive definite matrix A.

Define

$$
H_{\Gamma_{2}}^{1}(\Omega)=\left\{u \in H^{1}(\Omega)|u|_{\Gamma_{2}}=0\right\} .
$$

To obtain the stabilization of the system (2), we assume that the system (2) is well posed such that

$$
\begin{gathered}
u \in C^{1}\left([0,+\infty), L^{2}(\Omega)\right) \cap C\left([0,+\infty), H_{\Gamma_{2}}^{1}(\Omega)\right) \\
u_{t} \in C\left([0,+\infty), L^{2}\left(\Gamma_{1} \times(t, t-\tau(t))\right)\right) .
\end{gathered}
$$

The main result of this paper is the following.

Theorem 1. Let Assumption A hold true. Then, there exists a constant $C$, such that

$$
E(t) \leq C\left(\frac{E^{1 / p}(0)}{\left(t+\tau_{0}\right)^{1 / p}}+\frac{F\left(t+\tau_{0}\right)}{t+\tau_{0}} E(0)\right) \quad t>0 .
$$

Remark 2. If $p=1$ and $\phi(t)$ satisfies

$$
\phi_{1} \leq \phi(t) \leq \phi_{0} \quad \forall t \geq 0
$$


where $\phi_{0}$ and $\phi_{1}$ are positive constants, then it follows from (13) that there exist constants $T_{0}>0$ and $0<C_{0}<1$ such that

$$
E\left(T_{0}\right) \leq C_{0} E(0)
$$

Then, the decay of the energy $E(t)$ is exponential. Methods in $[21,22]$ are useful for Theorem 1 .

\section{Basic Inequality of the System}

In this section, we work on $\Omega$ with two metrics at the same time: the standard dot metric $\langle\cdot, \cdot\rangle$ and the Riemannian metric $g=\langle\cdot, \cdot\rangle_{g}$ given by (15).

If $f \in C^{1}\left(\mathbb{R}^{n}\right)$, we define the gradient $\nabla_{g} f$ of $f$ in the Riemannian metric $g$, via the Riesz representation theorem, by

$$
X(f)=\left\langle\nabla_{g} f, X\right\rangle_{g}
$$

where $X$ is any vector field on $\left(\mathbb{R}^{n}, g\right)$. The following lemma provides further relations between the two metrics; see [12], Lemma 3.

Lemma 3. Let $x=\left(x_{1}, \ldots, x_{n}\right)$ be the natural coordinate system in $\mathbb{R}^{n}$. Let $f, h$ be functions and let $\mathscr{H}, X$ be vector fields. Then,

(a)

$$
\langle H(x), A(x) X(x)\rangle_{g}=\langle(x), X(x)\rangle, \quad x \in \mathbb{R}^{n} ;
$$

(b)

$$
\nabla_{g} f=\sum_{i=1}^{n}\left(\sum_{j=1}^{n} a_{i j}(x) f_{x_{j}}\right) \frac{\partial}{\partial x_{i}}=A(x) \nabla f, \quad x \in \mathbb{R}^{n},
$$

where $\nabla f$ is the gradient of $f$ in the standard metric;

(c)

$$
\nabla_{g} f(h)=\left\langle\nabla_{g} f, \nabla_{g} h\right\rangle_{g}=\langle\nabla f, A(x) \nabla h\rangle, \quad x \in \mathbb{R}^{n},
$$

where the matrix $A(x)$ is given in formula (1).

To prove Theorem 1, we still further need several lemmas. Define

$$
E_{0}(t)=\frac{1}{2} \int_{\Omega}\left(u_{t}^{2}+\left|\nabla_{g} u\right|_{g}^{2}\right) d x
$$

Then, we have

$$
E(t)=E_{0}(t)+\xi \int_{t-\tau(t)}^{t} \int_{\Gamma_{1}} u_{t}^{2}(x, \rho) d \Gamma d \rho
$$

Lemma 4. Suppose that condition (14) holds true. Let $(u, w)$ be the solution of system (2). Then, there exist constants $C_{1}$, $C_{2}>0$ such that

$$
\begin{aligned}
& E(0)-E(T) \\
& \begin{aligned}
\geq C_{1} \int_{0}^{T} \int_{\Gamma_{1}} \phi(t)\left[u_{t}(x, t) g_{1}\left(u_{t}(x, t)\right)+u_{t}(x, t-\tau(t))\right. \\
\\
\left.\quad \times g_{1}\left(u_{t}(x, t-\tau(t))\right)\right] d \Gamma d t,
\end{aligned}
\end{aligned}
$$

where $T \geq 0$. Assertion (31) implies that $E(t)$ is decreasing.

Proof. Differentiating (13), we obtain

$$
\begin{aligned}
E^{\prime}(t)= & \int_{\Omega}\left(u_{t} u_{t t}+\nabla_{g} u \cdot \nabla u_{t}\right) d x \\
& +\int_{\Gamma_{1}} \xi \phi(t) u_{t}(x, t) g_{1}\left(u_{t}(x, t)\right) d \Gamma \\
& -\int_{\Gamma_{1}} \xi \phi(t-\tau(t))\left(1-\tau^{\prime}(t)\right) u_{t} \\
& \times(x, t-\tau(t)) g_{1}\left(u_{t}(x, t-\tau(t))\right) d \Gamma .
\end{aligned}
$$

Applying Green's formula and by integrating by parts with (3) and (8), we arrive at

$$
\begin{aligned}
E^{\prime}(t)= & \int_{\Gamma_{1}} \phi(t)\left[-\mu u_{t} g_{1}\left(u_{t}\right)-\lambda u_{t} g_{2}\left(u_{t}(x, t-\tau(t))\right)\right] d \Gamma \\
& +\int_{\Gamma_{1}} \xi \phi(t) u_{t}(x, t) g_{1}\left(u_{t}(x, t)\right) d \Gamma \\
& -\int_{\Gamma_{1}} \xi \phi(t-\tau(t))\left(1-\tau^{\prime}(t)\right) u_{t}(x, t-\tau(t)) g_{1} \\
& \left.\times\left(u_{t}(x, t-\tau(t))\right) d \Gamma \quad{ }^{2}{ }_{\Gamma_{1}} \phi(t)\left(-\mu u_{t} g_{1}\left(u_{t}\right)+\frac{|\lambda|}{2 \sqrt{1-d}} u_{t}^{2}+\xi u_{t} g_{1}\left(u_{t}\right)\right)\right) d \Gamma \\
& +\int_{\Gamma_{1}} \phi(t)\left[-c_{2}(1-d) \xi u_{t}(x, t-\tau(t)) g_{1}\right. \\
& \quad \times\left(u_{t}(x, t-\tau(t))\right) \\
& \left.+\frac{\sqrt{1-d}|\lambda|}{2} g_{2}^{2}\left(u_{t}(x, t-\tau(t))\right)\right] d \Gamma
\end{aligned}
$$

It follows from (3), (4), (12), and (14) that

$$
\begin{aligned}
E^{\prime}(t) \leq-C \phi(t) \int_{\Gamma_{1}}[ & u_{t}(x, t) g_{1}\left(u_{t}(x, t)\right) \\
& +u_{t}(x, t-\tau(t)) \\
& \left.\times g_{1}\left(u_{t}(x, t-\tau(t))\right)\right] d \Gamma,
\end{aligned}
$$

where $C>0$ satisfies

$$
C=\min \left\{\mu-\frac{|\lambda|}{2 \sqrt{1-d}}-\xi, c_{2}(1-d) \xi-\frac{\sqrt{1-d}|\lambda|}{2}\right\} .
$$

Then, inequality (31) follows directly from (34) integrating from 0 to $T$. 


\section{Proofs of Theorem 1}

From Proposition 2.1 in [12], we have the following identities.

Lemma 5. Suppose that $u(x, t)$ solves equation $u_{t t}+\mathscr{A} u=$ $0,(x, t) \in \Omega \times(0,+\infty)$ and that $\mathscr{H}$ is a vector field defined on $\bar{\Omega}$. Then, for $T \geq 0$,

$$
\begin{gathered}
\int_{0}^{T} \int_{\Gamma} \frac{\partial u}{\partial v_{\mathscr{A}}} \mathscr{H}(u) d \Gamma d t+\frac{1}{2} \int_{0}^{T} \int_{\Gamma}\left(u_{t}^{2}-\left|\nabla_{g} u\right|_{g}^{2}\right) \mathscr{H} \cdot v d \Gamma d t \\
=\left.\left(u_{t}, \mathscr{H}(u)\right)\right|_{0} ^{T}+\int_{0}^{T} \int_{\Omega} D_{g} \mathscr{H}\left(\nabla_{g} u, \nabla_{g} u\right) d x d t \\
+\frac{1}{2} \int_{0}^{T} \int_{\Omega}\left(u_{t}^{2}-\left|\nabla_{g} u\right|_{g}^{2}\right) \operatorname{div} \mathscr{H} d x d t .
\end{gathered}
$$

Moreover, assume that $P \in C^{1}(\bar{\Omega})$. Then,

$$
\begin{aligned}
\int_{0}^{T} & \int_{\Omega}\left(u_{t}^{2}-\left|\nabla_{g} u\right|_{g}^{2}\right) P d x d t \\
= & \left.\left(u_{t}, u P\right)\right|_{0} ^{T}+\frac{1}{2} \int_{0}^{T} \int_{\Omega} \nabla_{g} P\left(u^{2}\right) d x d t \\
& \quad-\int_{0}^{T} \int_{\Gamma} P u \frac{\partial u}{\partial \nu_{\mathscr{A}}} d \Gamma d t .
\end{aligned}
$$

Lemma 6. Suppose that all assumptions in Theorem 1 hold true. Let $u$ be the solution of the system (2). Then, there exists a positive constant $C$ for which

$$
\begin{aligned}
\int_{0}^{T} E(t) d t \leq & C E_{0}\left(T+\tau_{0}\right) \\
& +C \int_{0}^{T+\tau_{0}} \int_{\Gamma_{1}}\left(u_{t}^{2}+\left(\frac{\partial u}{\partial \nu_{\mathscr{A}}}\right)^{2}\right) d \Gamma d t \\
& +C \int_{0}^{T+\tau_{0}} \int_{\Gamma} \phi(t) u_{t}(x, t-\tau(t)) g_{1} \\
& \times\left(u_{t}(x, t-\tau(t))\right) d \Gamma d t
\end{aligned}
$$

where $T \geq 0$.

Proof. Let $\theta$ be a positive constant satisfying

$$
\frac{1}{2} \sup _{x \in \bar{\Omega}} \operatorname{div} H<\theta<\frac{1}{2} \inf _{x \in \bar{\Omega}} \operatorname{div} H+\rho_{0} .
$$

Set

$$
\mathscr{H}=H, \quad P=\theta-\rho_{0} .
$$

Substituting identity (37) into identity (36), we have

$$
\begin{aligned}
\Pi_{\Gamma}= & \left.\left(u_{t}, H(u)+P u\right)\right|_{0} ^{T} \\
& +\int_{0}^{T} \int_{\Omega}\left(D_{g} H\left(\nabla_{g} u, \nabla_{g} u\right)-\rho_{0}\left|\nabla_{g} u\right|_{g}^{2}\right) d x d t \\
& +\int_{0}^{T} \int_{\Omega}\left(\left(\frac{1}{2} \operatorname{div} H+\rho_{0}-\theta\right) u_{t}^{2}\right. \\
& \left.+\left(\theta-\frac{1}{2} \operatorname{div} H\right)\left|\nabla_{g} u\right|_{g}^{2}\right) d x d t,
\end{aligned}
$$

where

$$
\begin{aligned}
\Pi_{\Gamma}= & \int_{0}^{T} \int_{\Gamma} \frac{\partial u}{\partial v_{\mathscr{A}}}(H(u)+u P) d \Gamma d t \\
& +\frac{1}{2} \int_{0}^{T} \int_{\Gamma}\left(u_{t}^{2}-\left|\nabla_{g} u\right|_{g}^{2}\right) H \cdot v d \Gamma d t .
\end{aligned}
$$

We decompose $\Pi_{\Gamma}$ as

$$
\Pi_{\Gamma}=\Pi_{\Gamma_{1}}+\Pi_{\Gamma_{2}}
$$

Since $\left.u\right|_{\Gamma_{2}}=0$, we obtain $\left.\nabla_{\Gamma} u\right|_{\Gamma_{2}}=0$; that is,

$$
\nabla_{g} u=\frac{\partial u}{\partial v_{\mathscr{A}}} \frac{v_{\mathscr{A}}}{\left|v_{\mathscr{A}}\right|_{\mathscr{g}}^{2}} \quad \text { for } x \in \Gamma_{2}
$$

Similarly, we have

$$
H(u)=\left\langle H, \nabla_{g} u\right\rangle_{g}=\frac{\partial u}{\partial v_{\mathscr{A}}} \frac{H \cdot v}{\left|\nu_{\mathscr{A}}\right|_{g}^{2}} \quad \text { for } x \in \Gamma_{2} \text {. }
$$

Using formulas (44) and (45) in formula (42) on the portion $\Gamma_{2}$, with (19), we obtain

$$
\Pi_{\Gamma_{2}}=\frac{1}{2} \int_{0}^{T} \int_{\Gamma_{2}}\left(\frac{\partial u}{\partial \nu_{\mathscr{A}}}\right)^{2} \frac{H \cdot v}{\left|\nu_{\mathscr{A}}\right|_{g}^{2}} d \Gamma d t \leq 0
$$

From (19), we have

$$
\begin{aligned}
\Pi_{\Gamma_{1}}= & \int_{0}^{T} \int_{\Gamma_{1}} \frac{\partial u}{\partial v_{\mathscr{A}}}(H(u)+u P) d \Gamma d t \\
& +\frac{1}{2} \int_{0}^{T} \int_{\Gamma_{1}}\left(u_{t}^{2}-\left|\nabla_{g} u\right|_{g}^{2}\right) H \cdot v d \Gamma d t \\
\leq & C_{\varepsilon} \int_{0}^{T} \int_{\Gamma_{1}}\left(\frac{\partial u}{\partial v_{\mathscr{A}}}\right)^{2} d \Gamma d t+\varepsilon \int_{0}^{T} \int_{\Gamma_{1}}\left(u^{2}+\left|\nabla_{g} u\right|_{g}^{2}\right) d \Gamma d t \\
& +\int_{0}^{T} \int_{\Gamma_{1}}\left(C u_{t}^{2}-\delta\left|\nabla_{g} u\right|_{g}^{2}\right) d \Gamma d t \\
\leq & C \int_{0}^{T} \int_{\Gamma_{1}}\left(\frac{\partial u}{\partial \nu_{\mathscr{A}}}\right)^{2} d \Gamma d t+\varepsilon E_{0}(t)+C \int_{0}^{T} \int_{\Gamma_{1}} u_{t}^{2} d \Gamma d t .
\end{aligned}
$$

Substituting formulas (46) and (47) into formula (41), with (39), we obtain

$$
\begin{aligned}
\int_{0}^{T} E_{0}(t) d t \leq & C\left(E_{0}(0)+E_{0}(T)\right) \\
& +C \int_{0}^{T} \int_{\Gamma_{1}}\left(u_{t}^{2}+\left(\frac{\partial u}{\partial \nu_{\mathscr{A}}}\right)^{2}\right) d \Gamma d t
\end{aligned}
$$


Let $\varrho=\rho-\tau(\rho)$, and from (3), (7), (8), and (30), we have

$$
\begin{aligned}
& \int_{0}^{T+\tau_{0}} E_{0}(t) d t \\
& \quad+\int_{0}^{T+\tau_{0}} \int_{\Gamma} \phi(t) u_{t}(x, t-\tau(t)) g_{1} \times\left(u_{t}(x, t-\tau(t))\right) d \Gamma d t \\
& \geq \int_{0}^{T+\tau_{0}} E_{0}(t) d t+\frac{1}{\tau_{0}} \int_{0}^{T} \int_{t}^{t+\tau_{0}} \int_{\Gamma_{1}} \phi(\rho) u_{\rho} \\
& \quad \times(x, \rho-\tau(\rho)) g_{1}\left(u_{t}(x, \rho-\tau(\rho))\right) d \Gamma d \rho d t \\
& =\int_{0}^{T+\tau_{0}} E_{0}(t) d t+\frac{1}{\tau_{0}} \int_{0}^{T} \int_{t-\tau(t)}^{t+T_{1}-\tau\left(t+\tau_{0}\right)} \int_{\Gamma_{1}} \phi(\rho) u_{r} h o(x, \varrho) g_{1} \\
& \quad \times\left(u_{t}(x, \varrho)\right) d \Gamma \frac{d \varrho}{1-\tau^{\prime}(\rho)} d t \\
& \geq \int_{0}^{T+\tau_{0}} E_{0}(t) d t+\frac{c_{2}}{\left(1-d_{0}\right) \tau_{0}} \int_{0}^{T} \int_{t-\tau(t)}^{t} \int_{\Gamma_{1}} \phi(\varrho) u_{\varrho}(x, \varrho) g_{1} \\
& \quad \times\left(u_{t}(x, \varrho)\right) d \Gamma d \varrho d t \\
& \geq \min \left\{1, \frac{c_{2}}{\left(1-d_{0}\right) \tau_{0}}\right\} \int_{0}^{T} E(t) d t .
\end{aligned}
$$

Since

$$
\begin{aligned}
E_{0}(0) & =E_{0}\left(T+\tau_{0}\right)-\int_{0}^{T+\tau_{0}} \int_{\Gamma_{1}} u_{t} \frac{\partial u}{\partial v_{\mathscr{A}}} d \Gamma d t \\
& \leq E_{0}\left(T+\tau_{0}\right)+\frac{1}{2} \int_{0}^{T+\tau_{0}} \int_{\Gamma_{1}}\left(u_{t}^{2}+\frac{\partial u}{\partial v_{\mathscr{A}}}\right)^{2} d \Gamma d t,
\end{aligned}
$$

substituting formula (49) into formula (48), we obtain

$$
\begin{aligned}
& \int_{0}^{T} E(t) d t \\
& \leq C E_{0}\left(T+\tau_{0}\right)+C \int_{0}^{T+\tau_{0}} \int_{\Gamma_{1}}\left(u_{t}^{2}+\left(\frac{\partial u}{\partial v_{\mathscr{A}}}\right)^{2}\right) d \Gamma d t \\
& \quad+\int_{0}^{T+\tau_{0}} \int_{\Gamma} \phi(t) u_{t}(x, t-\tau(t)) g_{1} \\
& \quad \times\left(u_{t}(x, t-\tau(t))\right) d \Gamma d t .
\end{aligned}
$$

Inequality (38) holds.

Proof of Theorem 1. Since $E(t)$ is decreasing, from (38), for sufficiently large $T$, we have

$T E(T)$

$$
\begin{aligned}
\leq & C \int_{0}^{T+\tau_{0}} \int_{\Gamma_{1}}\left(u_{t}^{2}+\left(\frac{\partial u}{\partial v_{\mathscr{A}}}\right)^{2}\right) d \Gamma d t \\
& +C \int_{0}^{T+\tau_{0}} \int_{\Gamma} \phi(t) u_{t}(x, t-\tau(t)) g_{1}\left(u_{t}(x, t-\tau(t))\right) d \Gamma d t \\
\leq & C \int_{0}^{T+\tau_{0}} \int_{\Gamma_{1}}(\phi(t))^{2}\left[\left(g_{1}\left(u_{t}\right)\right)^{2}+\left(g_{2}\left(u_{t}\right)\right)^{2}\right] d \Gamma d t \\
& +C \int_{0}^{T+\tau_{0}} \int_{\Gamma_{1}} u_{t}^{2}(x, t) d \Gamma d t \\
& +C \int_{0}^{T+\tau_{0}} \int_{\Gamma} \phi(t) u_{t}(x, t-\tau(t)) g_{1}\left(u_{t}(x, t-\tau(t))\right) d \Gamma d t
\end{aligned}
$$

$$
\begin{aligned}
\leq & C \int_{0}^{T+\tau_{0}} \int_{\Gamma_{1}}(\phi(t))^{2}\left[\left(g_{1}\left(u_{t}\right)\right)^{2}+\left(g_{2}\left(u_{t}\right)\right)^{2}\right] d \Gamma d t \\
& +C F\left(T+\tau_{0}\right) \int_{0}^{T+\tau_{0}} \int_{\Gamma_{1}} \phi(t) u_{t}^{2}(x, t) d \Gamma d t \\
& +C \int_{0}^{T+\tau_{0}} \int_{\Gamma} \phi(t) u_{t}(x, t-\tau(t)) g_{1}\left(u_{t}(x, t-\tau(t))\right) d \Gamma d t,
\end{aligned}
$$

where $F(t)$ is defined in (8). With (4)-(8) and (31), we deduce that

$$
\begin{aligned}
T E & (T) \\
\leq & C \int_{0}^{T+\tau_{0}} \int_{\Gamma_{1}} \phi(t)\left(s^{2}+\left(g_{1}(s)\right)^{2}\right) d \Gamma d t \\
& +C F\left(T+\tau_{0}\right)\left(E(0)-E\left(T+\tau_{0}\right)\right) \\
\leq & C \int_{0}^{T+\tau_{0}} \int_{\left\{x \in \Gamma_{1}, u_{t}(x, t) \leq 1\right\}} \phi(t)\left(u_{t} g_{1}\left(u_{t}\right)\right)^{1 / p} d \Gamma d t \\
& +C F\left(T+\tau_{0}\right)\left(E(0)-E\left(T+\tau_{0}\right)\right) \\
\leq & C \int_{0}^{T+\tau_{0}} \int_{\Gamma_{1}} \phi(t)\left(u_{t} g_{1}\left(u_{t}\right)\right)^{1 / p} d \Gamma d t \\
& +C F\left(T+\tau_{0}\right)\left(E(0)-E\left(T+\tau_{0}\right)\right) \\
\leq & C \text { meas }\left(\Gamma_{1}\right) \int_{0}^{T+\tau_{0}} \phi(t) d t \\
& \cdot\left(\frac{1}{\operatorname{meas}\left(\Gamma_{1}\right) \int_{0}^{T+\tau_{0}} \phi(t) d t}\right. \\
& \left.\times \int_{0}^{T+\tau_{0}} \int_{\Gamma_{1}} \phi(t) u_{t} g\left(u_{t}\right) d \Gamma d t\right)^{1 / p} \\
& +C F\left(T+\tau_{0}\right)\left(E(0)-E\left(T+\tau_{0}\right)\right) \\
\leq & C\left(T+\tau_{0}\right)^{1-1 / p} \cdot\left(E(0)-E\left(T+\tau_{0}\right)\right)^{1 / p} \\
& +C F\left(T+\tau_{0}\right)\left(E(0)-E\left(T+\tau_{0}\right)\right) .
\end{aligned}
$$

Therefore,

$$
E(t) \leq C\left(\frac{E^{1 / p}(0)}{\left(T+\tau_{0}\right)^{1 / p}}+\frac{F\left(T+\tau_{0}\right)}{T+\tau_{0}} E(0)\right) .
$$

Note that $E(t)$ is decreasing; estimate (22) holds.

\section{Conflict of Interests}

The authors declare that there is no conflict of interests regarding the publication of this paper.

\section{Acknowledgments}

This research is supported by the National Science Foundation of China (nos. 91328201 and 41130422) and the National Basic Research Program of China (no. 2011CB201103). 


\section{References}

[1] A. Benaissa and A. Guesmia, "Energy decay for wave equations of $\phi$-Laplacian type with weakly nonlinear dissipation," Electronic Journal of Differential Equations, vol. 2008, no. 109, pp. $1-22,2008$.

[2] G. Chen, "Control and stabilization for the wave equation in a bounded domain," SIAM Journal on Control and Optimization, vol. 17, no. 1, pp. 66-81, 1979.

[3] G. Chen, "Control and stabilization for the wave equation in a bounded domain, part II," SIAM Journal on Control and Optimization, vol. 19, no. 1, pp. 114-122, 1981.

[4] A. Haraux, "Two remarks on dissipative hyperbolic problems," in Nonlinear Partial Differential Equations and Their Applications, vol. 122 of Research Notes in Mathematics, pp. 161-179, Pitman, Boston, Mass, USA, 1985.

[5] I. Lasiecka and D. Tataru, "Uniform boundary stabilization of semilinear wave equations with nonlinear boundary dampin," Differential Integral Equations, vol. 6, no. 3, pp. 507-533, 1993.

[6] K. Liu, "Locally distributed control and damping for the conservative systems," SIAM Journal on Control and Optimization, vol. 35, no. 5, pp. 1574-1590, 1997.

[7] E. Zuazua, "Exponential decay for the semilinear wave equation with locally distributed damping," Communications in Partial Differential Equations, vol. 15, no. 2, pp. 205-235, 1990.

[8] S. Feng and D. X. Feng, "Locally distributed control of wave equations with variable coefficients," Science in China A, vol. 44, no. 3, pp. 345-350, 2001.

[9] S. Nicaise and C. Pignotti, "Interior feedback stabilization of wave equations with time dependent delay," Electronic Journal of Differential Equations, vol. 2011, no. 41, pp. 1-20, 2011.

[10] S. Nicaise and C. Pignotti, "Stability and instability results of the wave equation with a delay term in the boundary or internal feedbacks," SIAM Journal on Control and Optimization, vol. 45, no. 5, pp. 1561-1585, 2006.

[11] S. Nicaise, C. Pignotti, and J. Valein, "Exponential stability of the wave equation with boundary time-varying delay," Discrete and Continuous Dynamical Systems S, vol. 4, no. 3, pp. 693-722, 2011.

[12] P.-F. Yao, "On the observability inequalities for exact controllability of wave equations with variable coefficients," SIAM Journal on Control and Optimization, vol. 37, no. 5, pp. 15681599, 1999.

[13] P.-F. Yao, "Observability inequalities for shallow shells," SIAM Journal on Control and Optimization, vol. 38, no. 6, pp. 17291756, 2000.

[14] P.-F. Yao, "Global smooth solutions for the quasilinear wave equation with boundary dissipation," Journal of Differential Equations, vol. 241, no. 1, pp. 62-93, 2007.

[15] P.-F. Yao, "Boundary controllability for the quasilinear wave equation," Applied Mathematics and Optimization, vol. 61, no. 2, pp. 191-233, 2010.

[16] P.-F. Yao, "Energy decay for the Cauchy problem of the linear wave equation of variable coefficients with dissipation," Chinese Annals of Mathematics B, vol. 31, no. 1, pp. 59-70, 2010.

[17] Z.-F. Zhang and P.-F. Yao, "Global smooth solutions of the quasilinear wave equation with internal velocity feedback," SIAM Journal on Control and Optimization, vol. 47, no. 4, pp. 20442077, 2008.

[18] I. Lasiecka, R. Triggiani, and P.-F. Yao, "Inverse/observability estimates for second-order hyperbolic equations with variable coefficients," Journal of Mathematical Analysis and Applications, vol. 235, no. 1, pp. 13-57, 1999.
[19] Z.-H. Ning and Q.-X. Yan, "Stabilization of the wave equation with variable coefficients and a delay in dissipative boundary feedback," Journal of Mathematical Analysis and Applications, vol. 367, no. 1, pp. 167-173, 2010.

[20] Z.-H. Ning, C.-X. Shen, and X. P. Zhao, "Stabilization of the wave equation with variable coefficients and a delay in dissipative internal feedback," Journal of Mathematical Analysis and Applications, vol. 405, no. 1, pp. 148-155, 2013.

[21] Z. H. Ning, C. X. Shen, X. P. Zhao, H. Li, C. S. Lin, and Y. M. Zhang, "Nonlinear boundary stabilization of the wave 5 equations with variable coeffcients and time dependent delay," Applied Mathematics and Computation, vol. 232, no. 1, pp. 511-520, 2014.

[22] S. Nicaise and C. Pignotti, "Stabilization of the wave equation with variable coefficients and boundary condition of memory type," Asymptotic Analysis, vol. 50, no. 1-2, pp. 31-67, 2006.

[23] P.-F. Yao, Modeling and Control in Vibrational and Structural Dynamics: A Differential Geometric Approach, Chapman \& Hall/ CRC Applied Mathematics \& Nonlinear Science, CRC Press, Boca Raton, Fla, USA, 2011.

[24] R. Gulliver, I. Lasiecka, W. Littman, and R. Triggiani, “The case for differential geometry in the control of single and coupled PDEs: the structural acoustic chamber," in Geometric Methods in Inverse Problems and PDE Control, vol. 137 of The IMA Volumes in Mathematics and Its Applications, pp. 73-181, Springer, New York, NY, USA, 2004. 


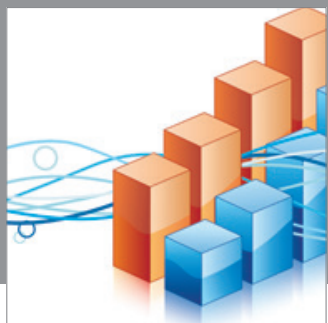

Advances in

Operations Research

mansans

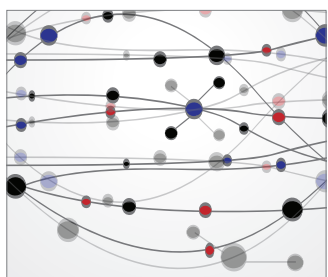

The Scientific World Journal
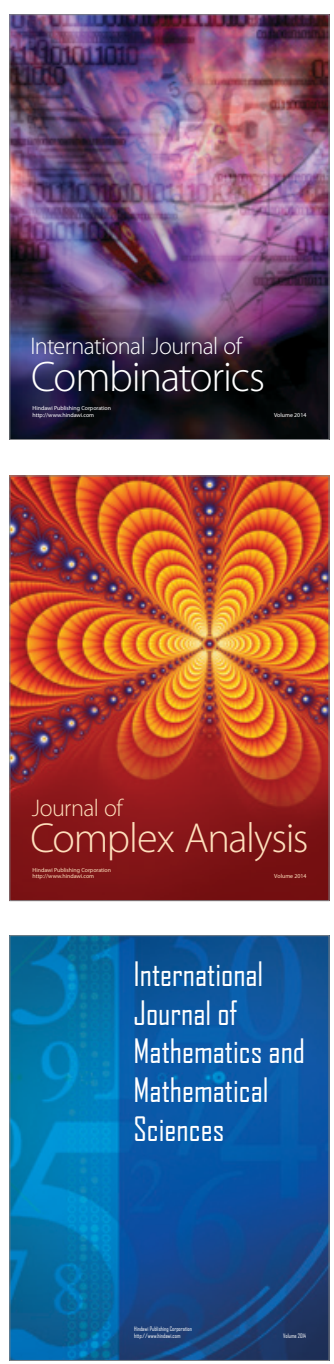
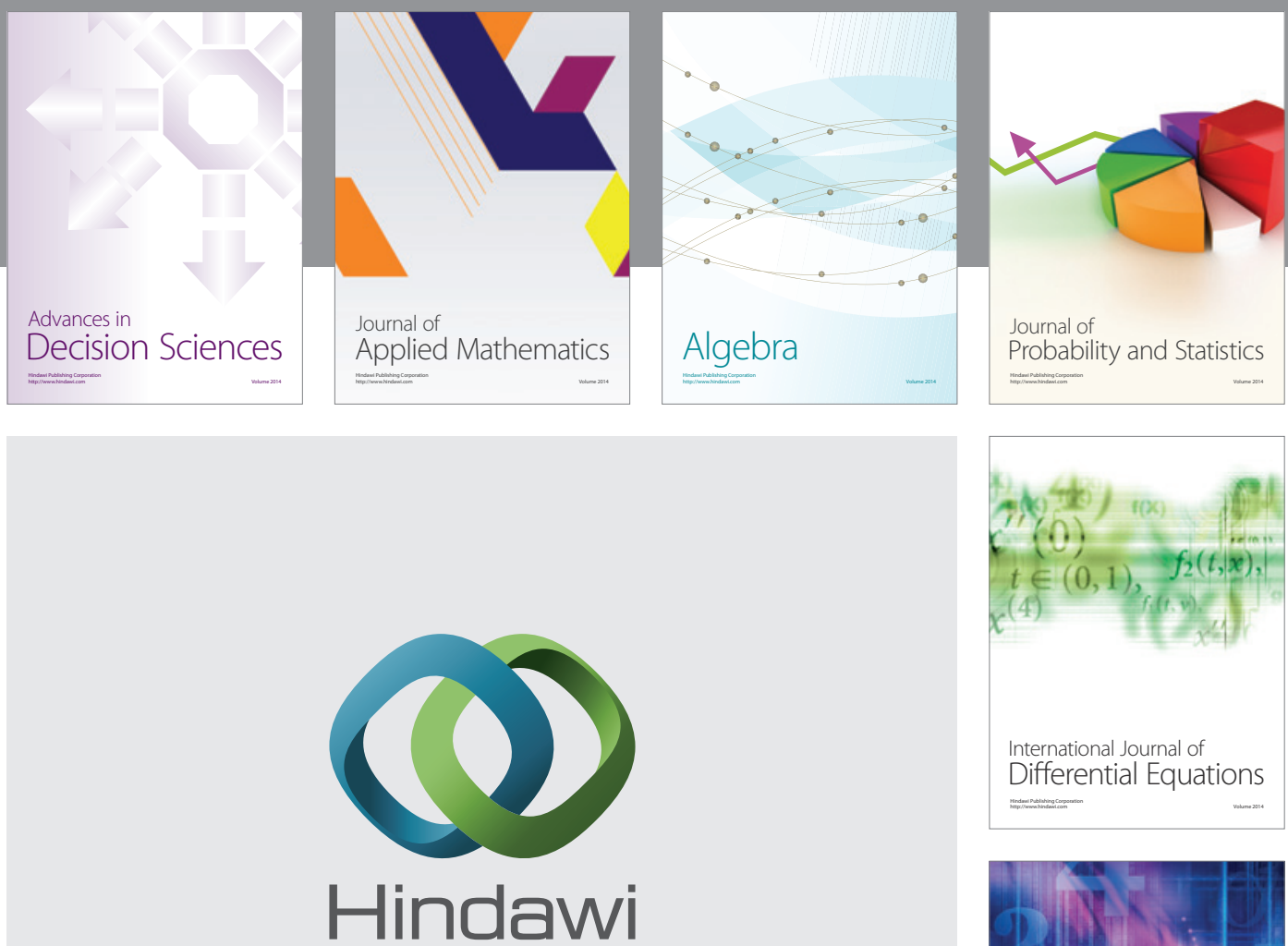

Submit your manuscripts at http://www.hindawi.com
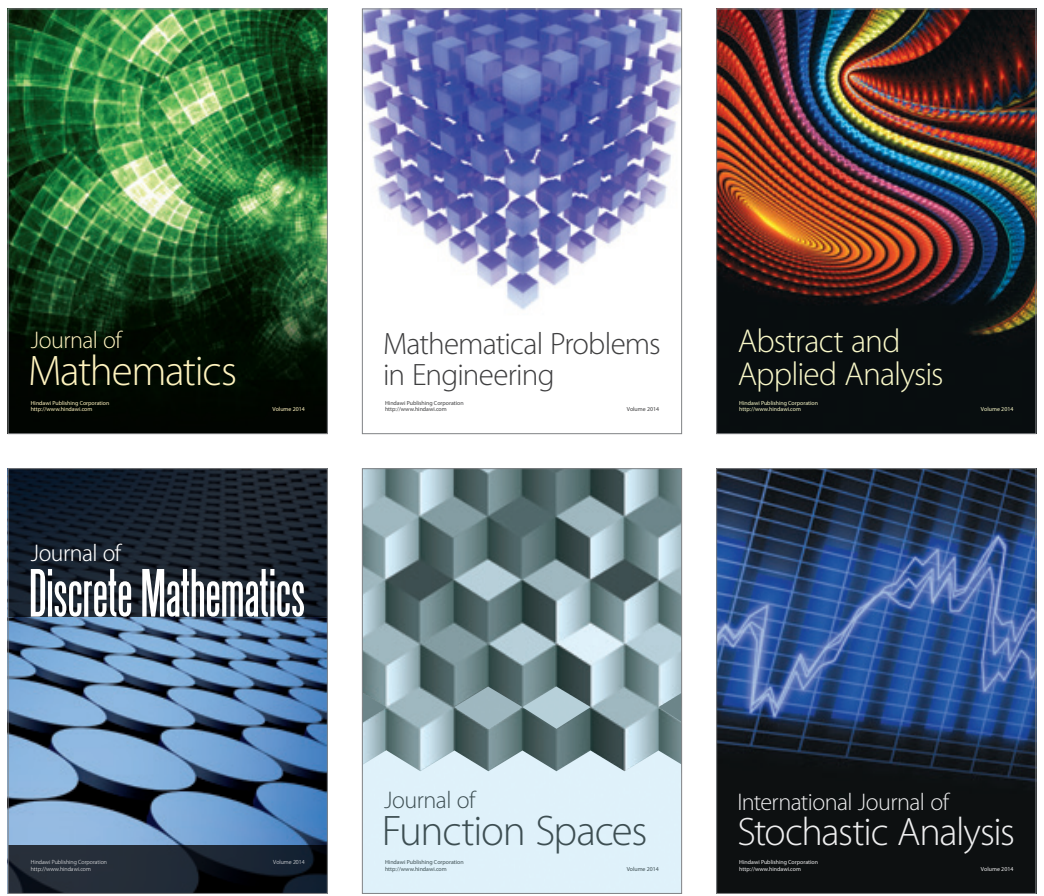

Journal of

Function Spaces

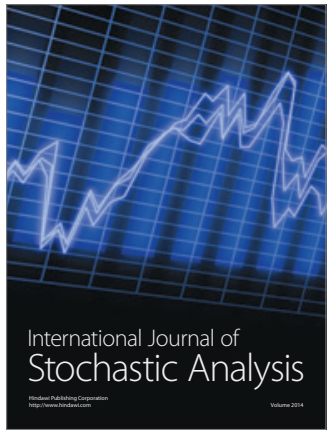

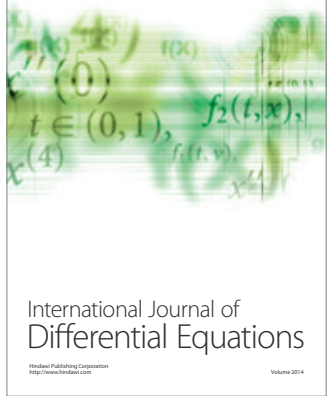
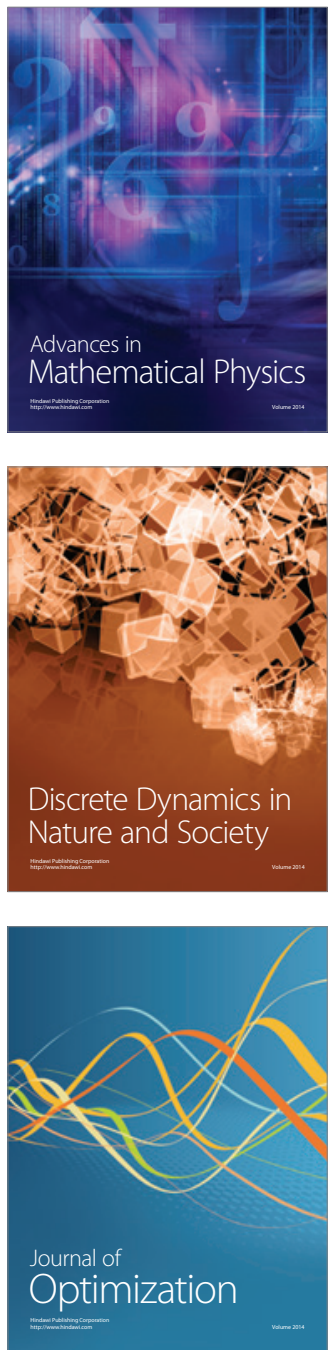